\title{
Scientific Programming in Computational Intelligence
}

\author{
Ricardo Soto, ${ }^{1}$ Eduardo Rodriguez-Tello, ${ }^{2}$ Stéphane Caro, ${ }^{3}$ and Frédéric Lardeux ${ }^{4}$ \\ ${ }^{1}$ Pontificia Universidad Católica de Valparaíso, 2362807 Valparaíso, Chile \\ ${ }^{2}$ CINVESTAV-Tamaulipas, Information Technology Laboratory, Km. 5.5 Carretera Victoria-Soto La Marina, \\ 87130 Victoria, TAMPS, Mexico \\ ${ }^{3}$ CNRS, Institut de Recherche en Communications et Cybernétique de Nantes, 44321 Nantes, France \\ ${ }^{4}$ LERIA, Université d'Angers, 49045 Angers, France \\ Correspondence should be addressed to Ricardo Soto; ricardo.soto@ucv.cl
}

Received 15 May 2016; Accepted 15 May 2016

Copyright (C) 2016 Ricardo Soto et al. This is an open access article distributed under the Creative Commons Attribution License, which permits unrestricted use, distribution, and reproduction in any medium, provided the original work is properly cited.

\section{Special Issue Overview}

Computational intelligence has been applied to a wide range of problems arising in different fields. Evolutionary programming, stochastic local search algorithms, swarm intelligence, and machine learning, among others, have been successfully applied to a range of optimization and classification problems which otherwise could not be tackled by traditional exact methods. More specifically, many problems arising in fields related to scientific programming have been increasingly addressed using metaheuristic algorithms from AI: scientific workflows scheduling/assignment, task assignment in distributed/parallel systems, (large-scale) data mining, knowledge discovery, and text analysis/process/classification, among others. This special issue is devoted to both theoretical and practical aspects of scientific programming related to computational intelligence.

\section{Summary of Included Papers}

This special issue includes six papers. The paper "Hybrid Multiple Soft-Sensor Models of Grinding Granularity Based on Cuckoo Searching Algorithm and Hysteresis Switching Strategy" proposes employing the cuckoo search metaheuristic for optimizing the performance parameters of a hysteresis switching strategy used to efficiently handle an interesting prediction problem from mineral concentration factories. The paper "A Matheuristic Approach Combining Local Search and Mathematical Programming" presents a hybrid approach based on local search and mathematical programming for efficiently solving the facility location problem. The local search algorithm is used to select a subset of promising facilities while mathematical programming is responsible for solving the subproblem to optimality. The third paper is called "AntStar: Enhancing Optimization Problems by Integrating an Ant System and A* Algorithm" which focuses on combining the well-known Ant System and A* Algorithm, the idea being to enhance the performance of the Ant System by inserting an evaluation function, the $\mathrm{A}^{*}$ algorithm, into the transition probability function of AS. Interesting experiments are performed on shortest-path problems. The fourth paper is named "Racing Sampling Based Microimmune Optimization Approach Solving Constrained Expected Value Programming" and presents a bioinspired microimmune optimization algorithm for solving a general kind of single-objective nonlinear constrained expected value programming. Comparative experiments illustrate excellent performance on higher-dimensional benchmark problems. The fifth paper is called "Efficient Parallel Sorting for Migrating Birds Optimization When Solving Machine-Part Cell Formation Problems" and describes the incorporation of parallel procedures into a relatively new metaheuristic called migrating birds optimization. The idea is to perform in parallel the several sorting tasks that this metaheuristic carries out when solving optimization problems. Interesting running time improvements are illustrated in the resolution of several machine part cell formation problem instances. Finally, the paper named "Profile Orientation and Slope 
Stability Analysis" presents an analysis of soil slope stability using a terrestrial laser scanner, particle swarm optimization, and the force equilibrium method. This study compares results obtained with those of previous studies making important observations.

\section{Acknowledgments}

We would like to thank the authors and the reviewers for making this special issue possible. Ricardo Soto would like to acknowledge support from CONICYT under the Grant CONICYT/FONDECYT/INICIACION/11130459.

Ricardo Soto

Eduardo Rodriguez-Tello

Stéphane Caro

Frédéric Lardeux 

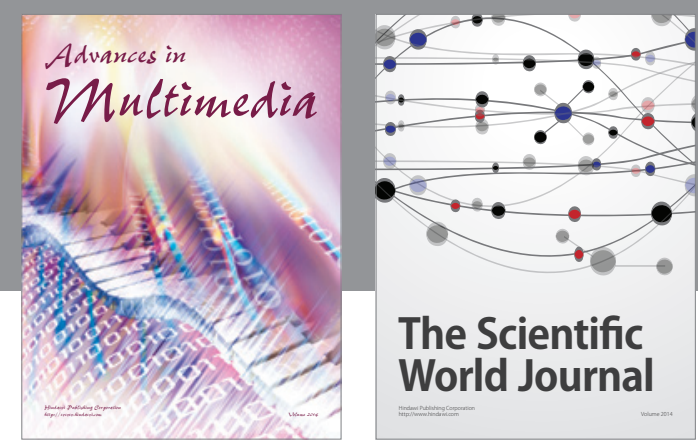

The Scientific World Journal
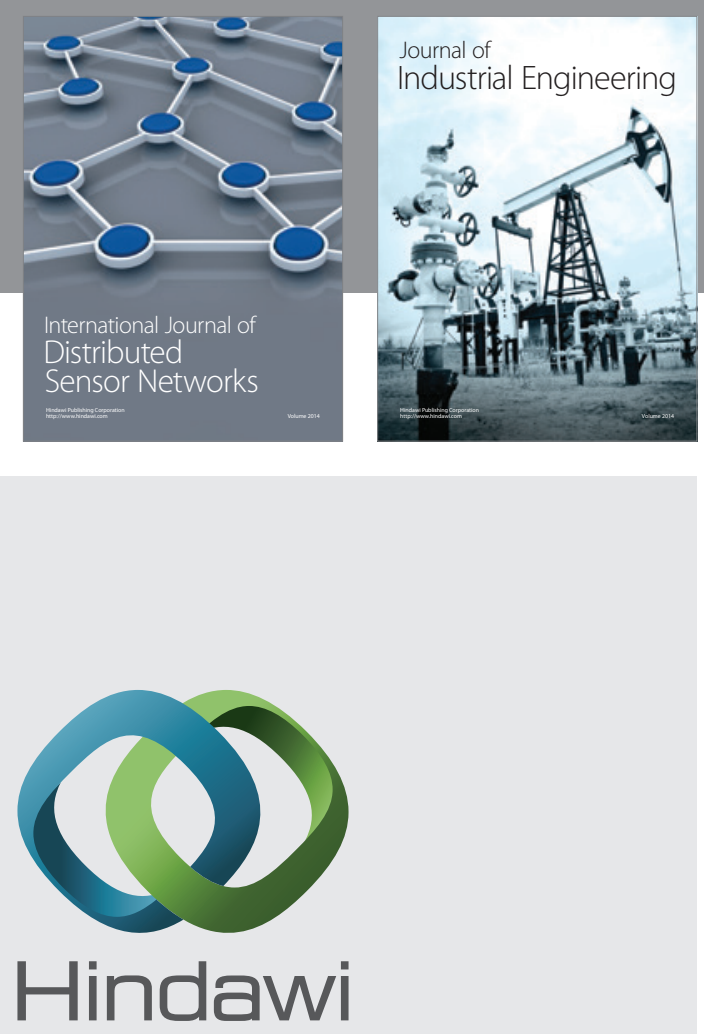

Submit your manuscripts at

http://www.hindawi.com

\section{Computer Networks} and Communications
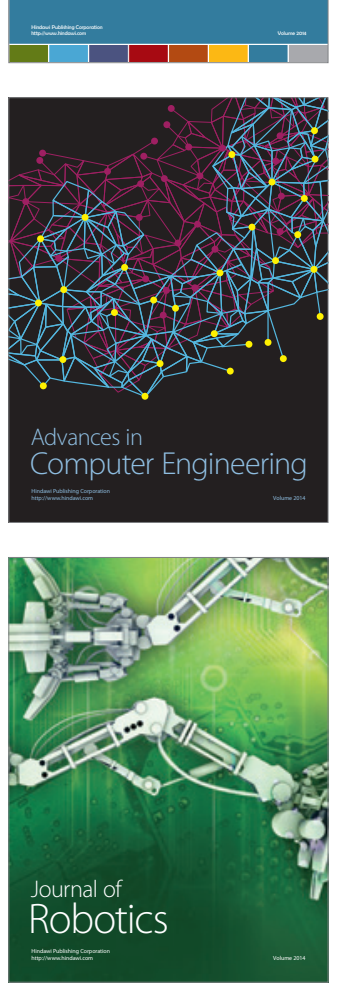
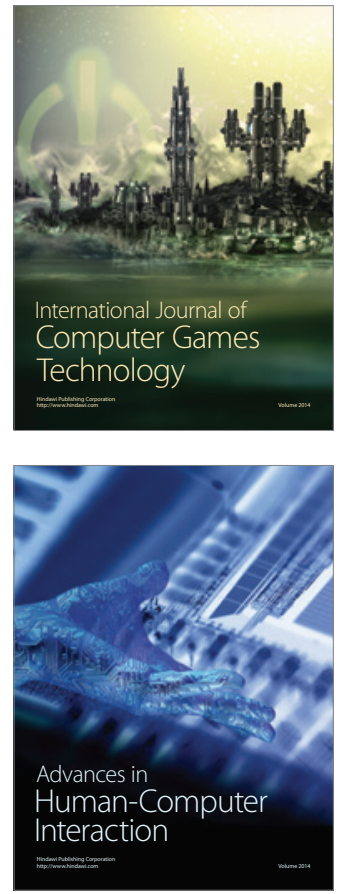
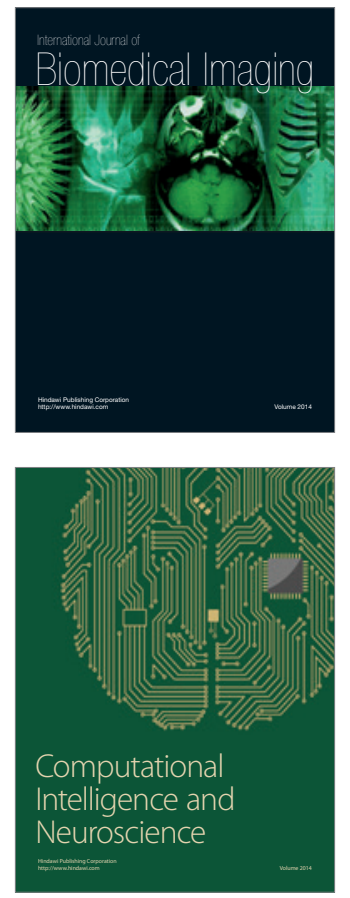
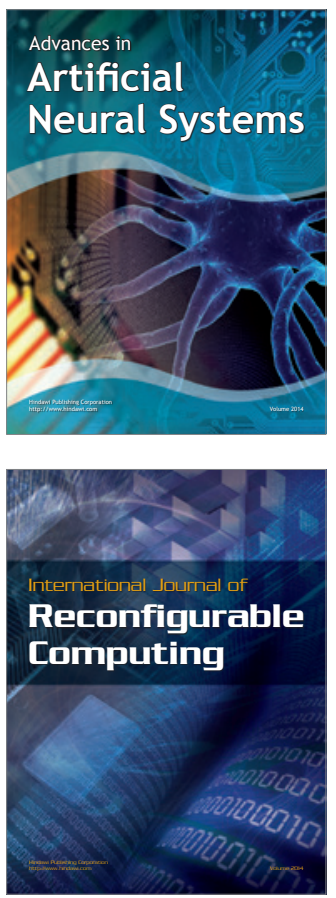
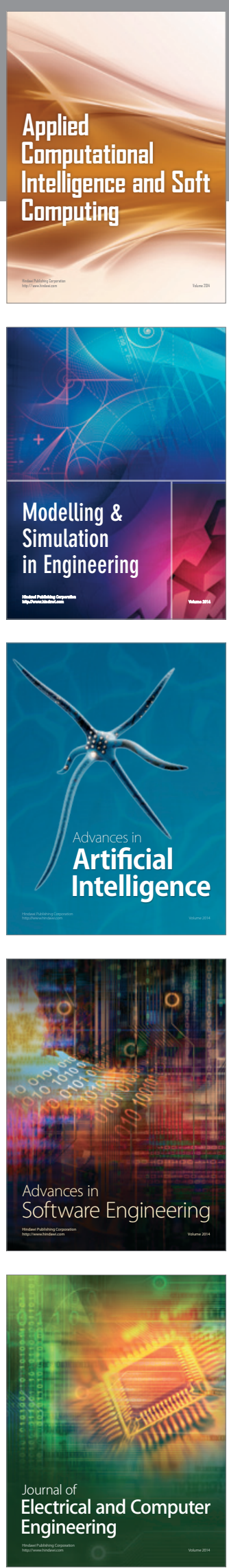